BIOINFORMATION

Discovery at the interface of physical and biologieal sciences

\section{Omics Metadata Management Software (OMMS)}

\author{
Martha O Perez-Arriaga ${ }^{1}$, Susan Wilson ${ }^{2}$, Kelly P Williams ${ }^{3}$, Joseph Schoeniger ${ }^{3}$, Russel L \\ Waymire 5 \& Amy Jo Powell2, 4,6*
}

1Department of Computer Science, Mail stop: MSC01 1130, 1 University of New Mexico, Albuquerque, NM 87131-0001; ${ }^{2}$ Center for Advanced Research Computing, University of New Mexico, Albuquerque, NM 87131; 3Systems Biology, Sandia National Laboratories, Mail Stop 9291, Livermore, CA 94550; "Department of Biology, Mail stop: MSC03 2020, 1 University of New Mexico, Albuquerque, NM 87131-0001; 5Security Systems Analysis, Sandia National Laboratories, Mail Stop 0757, Albuquerque, NM 87123; ${ }^{6}$ Computational Simulation Infrastructure, Sandia National Laboratories, Mail Stop 0845, Albuquerque, NM 87123; Amy Jo Powell - Email: ajpowel@sandia.gov; Phone: +1 505284 8050; *Corresponding author

Received October 11, 2014; Accepted March 16, 2015; Published April 30, 2015

\begin{abstract}
:
Next-generation sequencing projects have underappreciated information management tasks requiring detailed attention to specimen curation, nucleic acid sample preparation and sequence production methods required for downstream data processing, comparison, interpretation, sharing and reuse. The few existing metadata management tools for genome-based studies provide weak curatorial frameworks for experimentalists to store and manage idiosyncratic, project-specific information, typically offering no automation supporting unified naming and numbering conventions for sequencing production environments that routinely deal with hundreds, if not thousands of samples at a time. Moreover, existing tools are not readily interfaced with bioinformatics executables, (e.g., BLAST, Bowtie2, custom pipelines). Our application, the Omics Metadata Management Software (OMMS), answers both needs, empowering experimentalists to generate intuitive, consistent metadata, and perform analyses and information management tasks via an intuitive web-based interface. Several use cases with short-read sequence datasets are provided to validate installation and integrated function, and suggest possible methodological road maps for prospective users. Provided examples highlight possible OMMS workflows for metadata curation, multistep analyses, and results management and downloading. The OMMS can be implemented as a stand alone-package for individual laboratories, or can be configured for webbased deployment supporting geographically-dispersed projects. The OMMS was developed using an open-source software base, is flexible, extensible and easily installed and executed. The OMMS can be obtained at http://omms.sandia.gov.
\end{abstract}

Availability: The OMMS can be obtained at http://omms.sandia.gov.

Keywords: Bioinformatics, relational database management system, omics, next-generation sequencing, biological curation, opensource software, integrated workflow

Background:

Next-generation sequencing has revolutionized research and medicine, and has been accompanied by increasingly challenging, if underappreciated, metadata management requirements [1]. Sequencing projects that have large, complex metadata demand lightweight, flexible, stable software to support dataset standardization, biological curation activities, and streamlining of pre- and post- processing steps with pipeline analyses [2]. The few existing open-source tools for managing next-generation sequencing metadata have limited flexibility, thus hampering project-specific tailoring, and cannot be easily deployed and managed by a single administrator for multiple research sites $[3,4]$. Commerciallyavailable laboratory information management alternatives are typically expensive, cumbersome and require proprietary administration and maintenance for the software lifecycle [5], and none of the available tools, open-source or otherwise, readily integrate curation, processing and advanced analyses. 
We developed the Omics Metadata Management Software (OMMS), a flexible, extensible, open-source, web-based tool that provides semi-automated curation utilities, and integrated implementation with widely-used bioinformatics executables, such as BLAST [6] and Bowtie [7], for human-microbiomeoriented research in our laboratories [8]. Example use cases with publicly available human microbiome and chimpanzee RNASeq datasets $[9,10]$ are detailed to demonstrate OMMS function and versatility, and operation as a pipeline frontend.

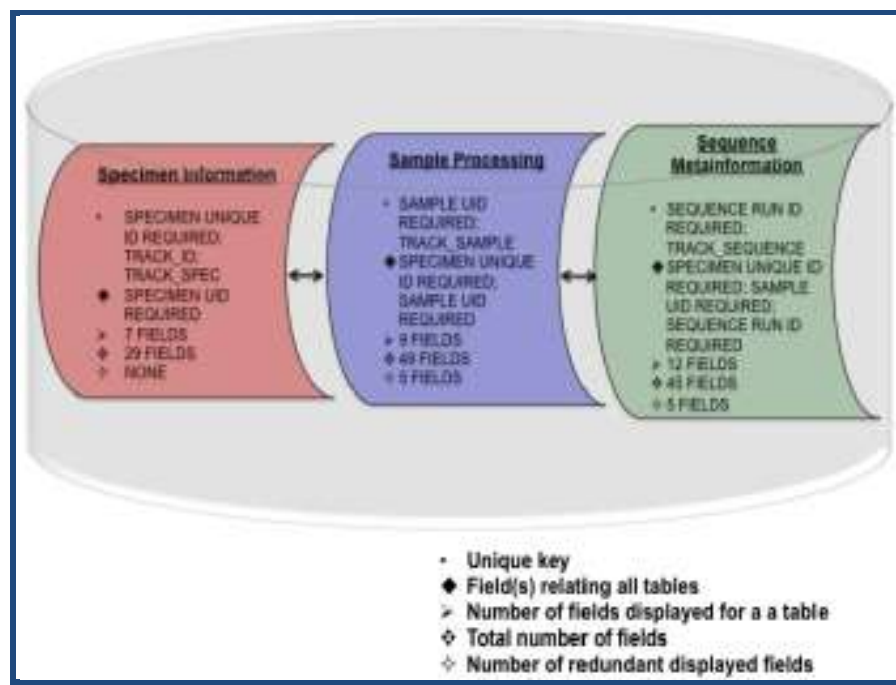

Figure 1: Omics Metadata Management Software. Core functionality resides in three tables, "Specimen Information," "Sample Processing," and "Sequence MetaInformation," which have fields with embedded automation supporting efficient data metadata entry, storage and intuitive entity relationships facilitating data sharing and analysis. These tables are accessed via the "MetaData" portal.

\section{Omics Metadata Management Software}

The OMMS was engineered to support a large, multidisciplinary, geographically-dispersed research team developing next-generation sequencing-based approaches for identifying potentially rare etiologic agents in human microbiomes across hundreds of distinct sample types [8]. The OMMS graphical user-interface (GUI) enables semi-automated project-specific metadata entries in each table (Figures $1 \&$ 2; Table 1, 3-5 (see supplementary material)), and associated input sequence data are referenced to archiving locations in directories generated on Linux-based file systems. Intuitive point-and-click selection of input sequence files, analysis configuration and execution are carried out in the "Analysis" portal (Figure 3; Table 2 (see supplementary material)). In examples provided here, BLAST, Bowtie 2, TopHat and Cufflinks were integrated and implemented with the OMMS interface [6, 7, 10], and in principle, any open-source application can be integrated with the OMMS, including inhouse pipelines, with custom scripts developed for that purpose.

\section{Methodology:}

\section{Creating a record}

The three main portals are displayed after login. Tables for detailed biological curation ("Specimen Info," "Sample Processing," "Sequence MetaInfo") reside in the "MetaData" portal. To create an entry, select "Create New," and then click ISSN 0973-2063 (online) 0973-8894 (print)

Bioinformation 11(4):165-172 (2015) on "New (Empty fields)" in the "Specimen Info" table, and provide required information (Figure 1; Table 1(see supplementary material)). The following (parentheses) were entered: Host Species (Homo sapiens); Tissue Sampled (Stool). Click the "Add Specimen" button to generate the "Specimen Unique ID" (HsStoo_01). For the second record, repeat the previous steps, but insert "Pan troglodyte" and "Brain" for the host and tissue, respectively, to generate "Specimen Unique ID" (PtBrai_02).

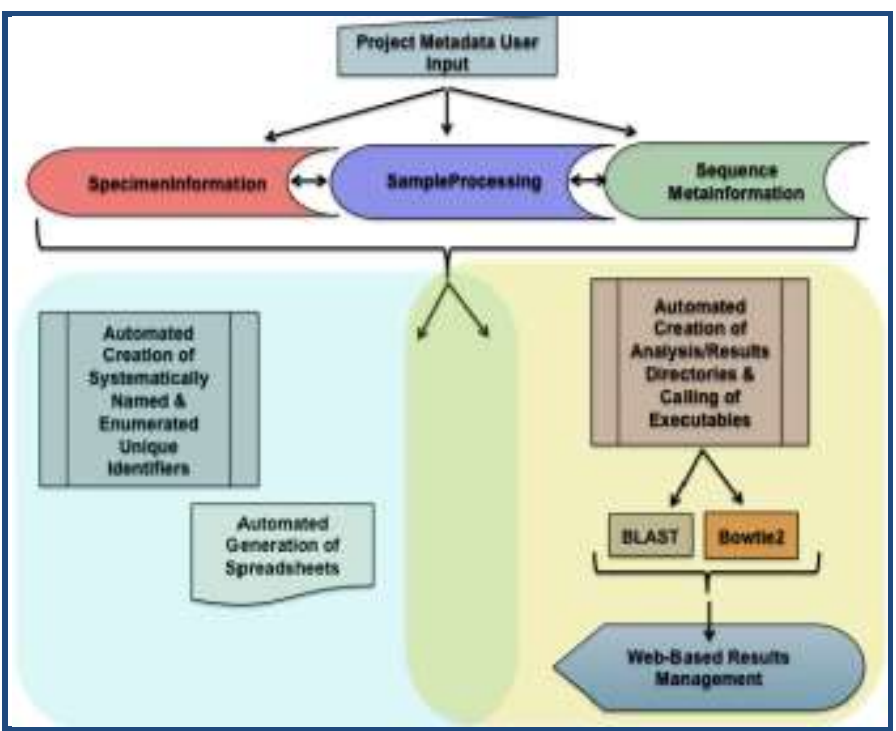

Figure 2: Unified framework for metadata management and state-of-the-art analyses. Curation (highlighted in aqua) and analyses (indicated in yellow) tasks are intrinsically related (overlap region) in next-generation sequencing studies, because sample handling and sequence production are multistep processes, and careful metadata tracking and management are required for downstream analyses and publication preparation. The OMMS supports user input of project metadata, automated creation of consistently named and enumerated unique identifiers for specimens, samples and sequence production information, and straightforward integration with bioinformatics utilities. Spreadsheets can be generated for structured data extraction and local download. Standard input and output of executables used here are stored in automatically-generated files and directories.

Corresponding records are generated in the "Sample Processing" table, with dropdown menus provided to streamline curation by explicitly linking the "Specimen Unique ID" (e.g., HsStoo_01, PtBrai_02) and the user-defined "Sample Alias" with the new sample entries. "Sample Unique ID" entries are generated by clicking "Add Sample" (e.g., HsStoo_01_01, PtBrai_02_01). Four corresponding records were created in the "Sequence MetaInfo" table to complete the curation exercise, and to illustrate functional integration with executables Table 2 (see supplementary material); in the "Provider Sequence Directory Name" field, arbitrary directory names were given; for "Fastq File Mate Pair 1" and "Fastq File Mate Pair 2," test input file names were used, and appropriate options were chosen for "Read Type" field for testing in this order: Bowtie 2 (single-, then paired end with human microbiome stool fastq files), BLAST (with human microbiome stool fasta input), Tophat and Cufflinks (with the single-end 
chimp RNASeq file). The "Sequence Run ID" and "Unique Experiment Name" were generated by clicking the "Add Sequence." In each of the tables, the "Update" function can be used to extend curation. Methods for generating and downloading custom metadata tables are further detailed in the "OMMS Integrated Workflow" link (under Quick Start).

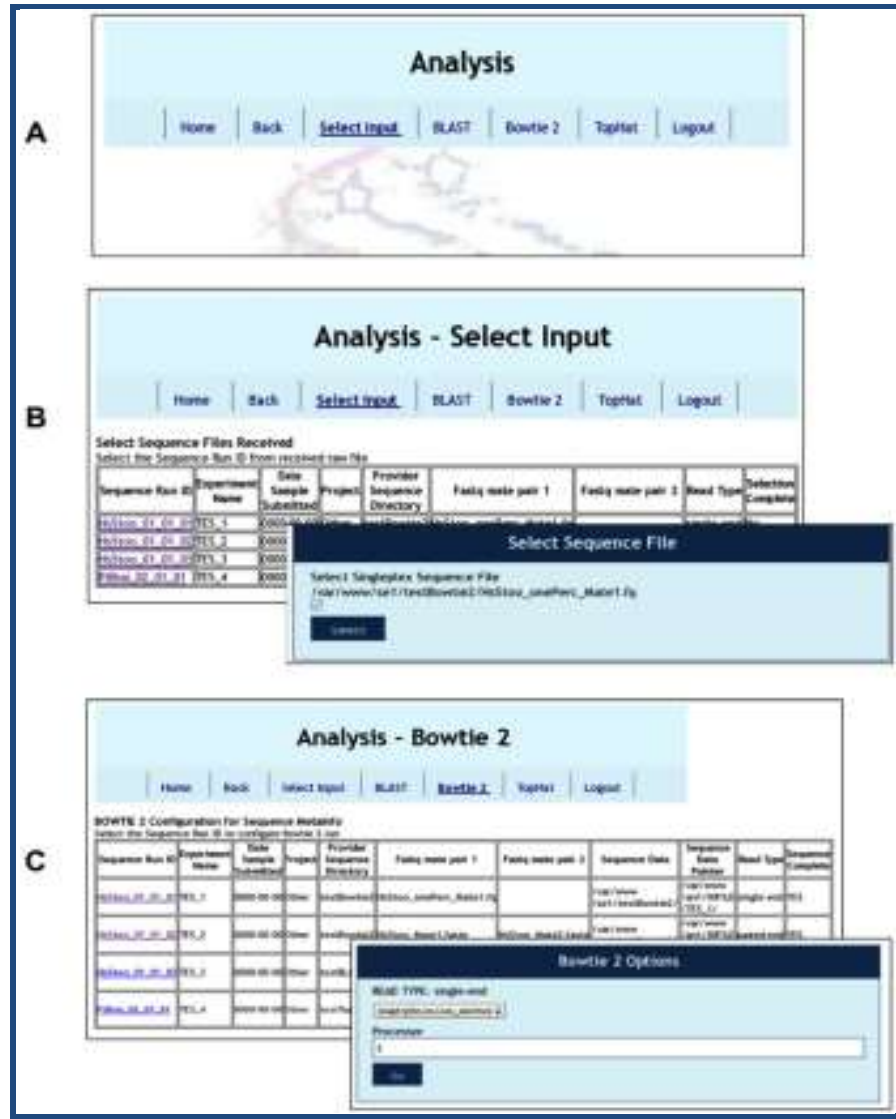

Figures 3A-C: Omics Metadata Management Software (OMMS) curation and analysis interface. The OMMS was designed to integrate and implement with open-source bioinformatics tools, such as BLAST, Bowtie 2 and Tophat and/or custom pipelines. These tools are accessed via the "Analysis Portal" (panel A). End users select the identifier ("Sequence Run ID") of interest, which is referenced to particular sequence files (panel B and inset). Following input selection, the desired program is chosen and parameterized (panel $\mathrm{C}$ and inset) to launch a run. Output from a given analysis run can be downloaded via the OMMS "Results" portal (not shown).

\section{Enabling integrated workflows}

To call integrated executables via the "Analysis" portal, click on "Select Input" for the relevant Sequence Run ID (e.g., HsStoo_01_01_01), and then choose the desired program (Figure 2). To launch a Bowtie 2 run on "Sequence Run ID" HsStoo_01_01_01, select the "Staphylococcus_aureus" index from the dropdown menu, and enter an integer value in "Processors" and click "Go." The results file name will appear, and standard output can be downloaded upon run completion. The same steps apply for paired-end analyses with Bowtie 2 . For BLAST runs, select the pertinent Sequence Run ID and input (HsStoo_BLAST500.fa), and choose the desired program (blastn) and database (Clostridium kluyveri). Set the significance threshold expectation (E) value at 0.001 or higher, and indicate the desired output format in the dropdown menu, and click "Go." Similar steps are followed for splice-variant and/or differential expression analyses using TopHat and Cufflinks (Figure 3; Table 2 (see supplementary material)). The chimp "Sequence Run ID" was selected (PtBrai_02_01_01, referenced to RNASeq file SRR023838_RNASeq.fq) and aligned with the hg19 index [7]. Standard output can be downloaded via the "Results" portal by choosing the "Sequence Run ID" of interest (e.g., HsStoo_01_01_01). The website provides additional instructions for building integrated analyses (in the “OMMS Integrated Workflow” link under Quick Start).

\section{Software:}

\section{Design and function}

Interoperable, open-source software packages (i.e., the LAMP bundle, Linux, Apache, MySQL, $\underline{\text { PHP) }}$ wereused to develop the browser-based OMMS interface to support next generation sequencing-based research efforts in our laboratories [8]. Realworld metadata associated with the test datasets were entered in the three tables, "Specimen Info," "Sample Processing," and "Sequence MetaInfo" (Figure 1; Table 1, 3-5 (see supplementary material)) to instantiate example database records. Most of the fields in the tables accommodate varied data types (e.g., the "Sample_Alias" field in the "Sample_Processing" table), such as character strings, but in cases with fewer possibilities, dropdown menus are provided (e.g., the "Nucleic Acid" field in the "Sample Processing" table).

Test datasets for validating installation and benchmarking integrated tools

Distinct hosts and tissues (Homo sapiens, Pan troglodyte; Stool, Brain) were used to demonstrate automated metadata tracking, storing and functional integration with utilities [9, 10]. Test datasets were obtained from the GenBank Short Read Archive (accessions SRX025177: SRR063480 and SRX008322: SRR023838), and were pre-processed using the NCBI SRA and Fastx Toolkits, and in-house custom scripts. These preprocessing steps are explained in the README file included in the distribution and in the "OMMS Integrated Workflows" link (see the Supplemental Materials for fine details pertaining to curation and pre-processing steps).

\section{Semi-automated curation and results downloading}

After entering the minimum required information (indicated by asterisks) for a specimen, the OMMS generates a unique identifier under the "Specimen_UID" field (Figure 1; Table 1 \& 3 (see supplementary material)) describing the subject/host and tissue/microhabitat from which nucleic acid preparations and sequence data will be derived (Figure 1 \& 2; Table 1 (see supplementary material)). Unique identifiers are automatically propagated to corresponding fields in the other tables (Figures 1 \& 2), intuitively linking specimen, sample and sequence data. Input sequence data files put can be uploaded, and results files (output) downloaded, as can metadata for specific entries, as well as table-overview custom spreadsheets (Figures 2 \& 3 ).

\section{Concluding Remarks:}

The freeware reported here guarantees standardized, intelligible, automated curation and management of biological 
metadata, and supports integrated analyses. Recent events, from the outbreak of Ebola Virus Disease in West Africa, to the emergence of antibiotic-resistant bacteria (e.g., Clostridium difficile, Carbapenem-resistant Enterobacteriaceae), make it impossible to overstate the importance of rigorous metadata curation and management systems in high-intensity scenarios, clinical and otherwise. For our project, the OMMS frontend was foundational for handling metadata inherent to nextgeneration sequencing-based experiments involving large numbers of samples at a time. In the context of a host microbiome, potential etiologic agents are typically rare and difficult to detect using standard in silico and experimental approaches, and careful metadata curation is crucial for identifying signal (infectious disease) in the presence of overwhelming noise (background microbiota) and results interpretation. Looking ahead, the OMMS and OMMS-user tailored versions will represent easy-to-use promising tools for addressing microbiome-centric research, from clinical and public health challenges, to exploring new frontiers in agricultural research and development, where handing and tracking hundreds, if not thousands, of samples from diverse subjects at a particular location and time are necessary. Additionally, the OMMS enables development of integrated workflows with state-of-the-art utilities (Blast, Bowtie 2) and in-house pipelines (e.g., local implementations of Galaxy), facilitating fine-grained comparative analyses, such as strain discrimination (e.g., Zaire vs. Sudan ebolavirus) and microbiome composition and functional profiling.

\section{Acknowledgement:}

This R \& D was supported by the Laboratory Directed Research and Development (LDRD) program at Sandia National Laboratories [under the auspices of the Rapid Threat Organism Recognition (RapTOR) Grand Challenge (LDRD \# 142042)]. Sandia National Laboratories are multi-program laboratories managed and operated by Sandia Corporation, a wholly-owned subsidiary of Lockheed Martin Corporation, for the U.S. Department of Energy's National Nuclear Security Administration (contract DE-AC04-94AL85000). We offer our sincerest thanks to the RapTOR team, and Dr. Elisa LaBauve, in particular, for invaluable discussions around software design and development, and Professor Melanie Moses for guidance on manuscript preparation. As ever, I extend my deepest gratitude to Professors Donald O. Natvig and Gavin C. Conant for invaluable, ongoing scientific discussions, editorial assistance and software testing. Sincerest thanks to Steven Arroyo, Brian Nelson, Michael W. Folsom and Mark D. Murton for software testing and website development. We are grateful to the University of New Mexico Center for Advanced Research Computing and Dr. Susan Atlas, Director, for computational resources and technical assistance provided in support of this work.

References:

[1] Pareek C et al. J Appl Genetics 2011 52: 413 [PMID: 21698376]

[2] Wruck W et al. Brief Bioinform 2014 15: 65 [PMID: 23047157]

[3] Rocca-Serra P et al. Bioinformatics 2010 26: 2354 [PMID: 20679334]

[4] Wolstencroft $\mathrm{K}$ et al. Bioinformatics 2011 27: 2021 [PMID: 21622664]

[5] Gross J Labguru 2011, http://www.labguru.com/, TelAviv, Israel: BioData Ltd

[6] Altschul SF et al. Nucleic Acids Research 1997 25: 3389 [PMID: 9254694]

[7] Langmead B \& Salzberg SL, Nat Meth 2012 9: 357 [PMID: 22388286]

[8] Bent ZW et al. Anal Biochem. 2013 438: 90 [PMID: 23535274]

[9] Human Microbiome Project Consortium. Nature 2012 486: 207 [PMID: 22699609]

[10] Trapnell C et al. Nat Protocols. 2012 7: 562 [PMID 22383036]
Edited by $\mathbf{P}$ Kangueane

Citation: Arriaga et al. Bioinformation 11(4): 165-172 (2015)

License statement: This is an open-access article, which permits unrestricted use, distribution, and reproduction in any medium, for non-commercial purposes, provided the original author and source are credited 


\section{Supplementary material:}

Table 1: Overview of tables with embedded automation supporting specimen, sample and sequence data curation and storage. The OMMS supports next-generation sequencing projects, which invariably have substantial metadata management overhead. Core software functionality resides in the three interoperable tables, which are accessed via the "MetaData" portal.

\begin{tabular}{|c|c|c|c|c|c|c|}
\hline Table Name & $\begin{array}{l}\text { Total } \\
\text { number of } \\
\text { fields }\end{array}$ & $\begin{array}{l}\text { Number of } \\
\text { required } \\
\text { fields }\end{array}$ & $\begin{array}{l}\text { Number of fields } \\
\text { present in user } \\
\text { interface "Consult" } \\
\text { mode }\end{array}$ & $\begin{array}{l}\text { Number of } \\
\text { redundant } \\
\text { fields }\end{array}$ & $\begin{array}{l}\text { Number of } \\
\text { automated } \\
\text { fields }\end{array}$ & $\begin{array}{l}\text { Fields used to relate other } \\
\text { tables }\end{array}$ \\
\hline $\begin{array}{l}\text { Specimen } \\
\text { Info }\end{array}$ & 29 & 6 & 7 & 0 & 7 & Specimen_UID_req \\
\hline $\begin{array}{l}\text { Sample } \\
\text { Processing }\end{array}$ & 48 & 5 & 9 & 5 & 7 & $\begin{array}{l}\text { Specimen_UID_req } \\
\text { Sample_UID_req }\end{array}$ \\
\hline $\begin{array}{l}\text { Sequence } \\
\text { MetaInfo }\end{array}$ & 44 & 6 & 12 & 5 & 9 & $\begin{array}{l}\text { Specimen_UID_req } \\
\text { Sample_UID_req } \\
\text { SequenceRun_ID_req }\end{array}$ \\
\hline
\end{tabular}

Table 2: Integration of curation tasks and bioinformatics analyses. The "Omics Metadata Management System" (OMMS) is designed for integrated use with Bowtie 2, Tophat, Cufflinks and BLAST, and can be extended and configured to call and run other open-source executables, such as

\begin{tabular}{|c|c|c|c|c|c|c|c|c|c|}
\hline $\begin{array}{l}\text { Specimen } \\
\text { Unique ID }\end{array}$ & $\begin{array}{l}\text { Sample } \\
\text { Unique } \\
\text { ID }\end{array}$ & \begin{tabular}{ll}
\multicolumn{2}{l}{ Sequenc } \\
e $\quad$ Run \\
ID
\end{tabular} & $\begin{array}{l}\text { Provider } \\
\text { Sequence } \\
\text { Directory Name }^{3}\end{array}$ & $\begin{array}{l}\text { Unique } \\
\text { Experiment } \\
\text { Name }^{3}\end{array}$ & $\begin{array}{l}\text { Sequence } \\
\text { Input (Mate } \\
\text { Pair 1) }\end{array}$ & $\begin{array}{l}\text { Sequence Input } \\
\text { (Mate Pair 2) }^{3}\end{array}$ & Executable & Input Name ${ }^{3}$ & $\begin{array}{l}\text { Output } \\
\text { Name }^{3}\end{array}$ \\
\hline HsStoo_01 & $\begin{array}{l}\text { HsStoo_ } \\
01 \_01\end{array}$ & $\begin{array}{l}\text { HsStoo_- } \\
01 \_01 \_0 \\
1\end{array}$ & testBowtie2 & TES_1 & $\begin{array}{l}\text { HsStoo_one } \\
\text { Perc_Mate1. } \\
\text { fq }\end{array}$ & & bowtie2 & $\begin{array}{l}\text { INFILE/TES_ } \\
1 / \text { TES_1 }\end{array}$ & $\begin{array}{l}\text { OUTFILE/T } \\
\text { ES_1/TES_1 } \\
-1 . \text { sam }\end{array}$ \\
\hline HsStoo_01 & $\begin{array}{l}\text { HsStoo_ } \\
01 \_01\end{array}$ & $\begin{array}{l}\text { HsStoo_- } \\
01 \_01 \_0 \\
2\end{array}$ & testBowtie2 & TES_2 & $\begin{array}{l}\text { HsStoo_Mat } \\
\text { e1.fq }\end{array}$ & HsStoo_Mate2.fq & bowtie2 & $\begin{array}{l}\text { INFILE/TES_- } \\
\text { 2/PAIR1/TES } \\
2 \\
\text { INFILE/TES_- } \\
\text { 2/PAIR2/TES } \\
2\end{array}$ & $\begin{array}{l}\text { OUTFILE/T } \\
\text { ES_2/PAIR1 } \\
\text { /TES_2- } \\
\text { 1.sam }\end{array}$ \\
\hline HsStoo_01 & $\begin{array}{l}\text { HsStoo_ } \\
01 \_01\end{array}$ & $\begin{array}{l}\text { HsStoo_- } \\
01 \_01 \_0 \\
3\end{array}$ & testBLAST & TES_3 & $\begin{array}{l}\text { HsStoo_BL } \\
\text { AST500.fa }\end{array}$ & & blastn & $\begin{array}{l}\text { INFILE/TES_ } \\
\text { 3/TES_3 }\end{array}$ & $\begin{array}{l}\text { OUTFILE/T } \\
\text { ES_3/TES_3 } \\
-1\end{array}$ \\
\hline HsBrai_02 & $\begin{array}{l}\text { HsBrai_- } \\
02 \_01\end{array}$ & $\begin{array}{l}\text { HsBrai_ } \\
02 \_01 \_0 \\
1\end{array}$ & testTopCuff & TES_4 & $\begin{array}{l}\text { SRR023838_ } \\
\text { RNASeq.fq }\end{array}$ & & $\begin{array}{l}\text { tophat } \\
\text { cufflinks }\end{array}$ & $\begin{array}{l}\text { INFILE/TES_ } \\
\text { 4/TES_4 }\end{array}$ & $\begin{array}{l}\text { OUTFILE/T } \\
\text { ES_4/TES_4 } \\
- \\
\text { 1/tophat_out/ } \\
\text { accepted_hit } \\
\text { s.bam }\end{array}$ \\
\hline
\end{tabular}

custom pipelines. Sequence files are referenced to automatically generated metadata entries (e.g., "Sample Unique ID", "Sequence Run ID"), and these files can be used as input for programs accessed via the "Analysis" portal. The OMMS also generates directories to store results (output) for each run of a program. Output can be accessed and downloaded via the "Results" portal.

Table 3: Specimen Information

\begin{tabular}{|c|c|c|c|c|}
\hline Field Name & Data Type & Required Field & Automated Field & Drop-down Menu \\
\hline Specimen_UID_req & varchar(100) & & $X$ & \\
\hline Specimen_Alias_req & varchar(100) & & & \\
\hline Date_Acquired_req & date & & & \\
\hline Acquired_By_req & $\operatorname{varchar}(100)$ & $x$ & & \\
\hline Storage_Location_req & varchar(100) & & & \\
\hline Source_req & varchar(100) & $X$ & & \\
\hline Specimen_Type_req & varchar(100) & $x$ & & $X$ \\
\hline Host_ID_req & varchar(100) & $x$ & & \\
\hline Host_Organism_req & $\operatorname{varchar}(100)$ & $x$ & & \\
\hline Host_Ploidy & varchar(100) & & & \\
\hline
\end{tabular}




\begin{tabular}{|c|c|c|c|c|}
\hline Host_Gender & $\operatorname{varchar}(100)$ & & & $x$ \\
\hline Host_Age & $\operatorname{int}(11)$ & & & \\
\hline Host_Ethnicity & varchar(100) & & & \\
\hline Tissue_Sampled_req & $\operatorname{varchar}(100)$ & $X$ & & \\
\hline Diagnostic_Screen & varchar(5000) & & & \\
\hline Pathogen_Type & $\operatorname{varchar}(100)$ & & & \\
\hline Pathogen_Dose & varchar(100) & & & \\
\hline Pathogen_Delivery & varchar(100) & & & \\
\hline $\begin{array}{l}\text { Specimen_Collection_Tim } \\
\text { ing }\end{array}$ & $\operatorname{varchar}(100)$ & & & \\
\hline $\begin{array}{l}\text { Experimentalists_notes_u } \\
\text { pload }\end{array}$ & varchar(100) & & & \\
\hline fileuploaded & mediumblob & & & \\
\hline filetype & filetype & & & \\
\hline Remarks & varchar(5000) & & & \\
\hline createdby & $\operatorname{varchar}(20)$ & & $X$ & \\
\hline timecreation & timestamp & & $x$ & \\
\hline modifiedby & varchar(20) & & $x$ & \\
\hline timemodified & timestamp & & $X$ & \\
\hline track_id & $\operatorname{int}(11)$ & & $X$ & \\
\hline track_spec & $\operatorname{int}(11)$ & & $X$ & \\
\hline
\end{tabular}

Table 4: Sample Processing

\begin{tabular}{|c|c|c|c|c|}
\hline Field Name & Data Type & Required Field & Automated Field & Drop-down Menu \\
\hline Specimen_UID_req & $\operatorname{varchar}(100)$ & $X$ & & $X$ \\
\hline Specimen_UID_sec & $\operatorname{varchar}(50)$ & & & \\
\hline Sample_UID_req & $\operatorname{varchar}(100)$ & & $X$ & \\
\hline Sample_Alias_req & $\operatorname{varchar}(100)$ & & & \\
\hline Nucleic_Acid_req & $\operatorname{varchar}(100)$ & & & \\
\hline Target_Nucleic_Acid_req & $\operatorname{varchar}(100)$ & & & \\
\hline Purification_Nucleic_Acid_Method_req & $\operatorname{varchar}(100)$ & $X$ & & $x$ \\
\hline Purified_By_req & $\operatorname{varchar}(100)$ & $x$ & & \\
\hline Date_Nucleic_Acid_Purified_req & date & & & \\
\hline Purified_Nucleic_Acid_Yield_Nanograms & float $(7,2)$ & & & \\
\hline QC_Result & $\operatorname{varchar}(5000)$ & & & \\
\hline Nucleic_Acid_Modification_Method & $\operatorname{varchar}(100)$ & & & $x$ \\
\hline Nucleic_Acid_Modified_By & $\operatorname{varchar}(100)$ & & & \\
\hline Date_Nucleic_Acid_Modified & date & & & \\
\hline Modified_Nucleic_Acid_Type & $\operatorname{varchar}(100)$ & & & \\
\hline Modified_Nucleic_Acid_Yield_Nanograms & float $(7,2)$ & & & \\
\hline Modified_Nucleic_Acid_QC_Result & varchar(5000) & & & \\
\hline Suppression_Product_Name & $\operatorname{varchar}(100)$ & & & \\
\hline Suppression_Method & $\operatorname{varchar}(100)$ & & & $X$ \\
\hline Suppressed_By & $\operatorname{varchar}(100)$ & & & \\
\hline Date_Nucleic_Acid_Suppressed & date & & & \\
\hline Suppressed_Nucleic_Acid_Yield_Nanograms & float $(7,2)$ & & & \\
\hline
\end{tabular}




\begin{tabular}{|c|c|c|c|}
\hline Suppressed_Nucleic_Acid_QC_Result & $\operatorname{varchar}(5000)$ & & \\
\hline Barcode & $\operatorname{varchar}(200)$ & $x$ & \\
\hline Primer_Set & varchar(30) & $x$ & \\
\hline Primer_Name & $\operatorname{varchar}(100)$ & & \\
\hline Barcode_Type & $\operatorname{varchar}(20)$ & & \\
\hline Library_Preparation_ID_req & $\operatorname{varchar}(1000)$ & & \\
\hline Library_Preparation_Method_req & $\operatorname{varchar}(100)$ & & \\
\hline Library_Prepared_By_req & $\operatorname{varchar}(100)$ & & \\
\hline Date_Library_Prepared_req & date & & \\
\hline Library_Nucleic_Acid_Input_Nanograms_req & float $(7,2)$ & & \\
\hline Library_Prep_Final_Volume_Microliters_req & float $(7,2)$ & & \\
\hline $\begin{array}{l}\text { Final_Library_Concentration_Nanograms_Micro } \\
\text { liter_req }\end{array}$ & float $(7,2)$ & & \\
\hline Library_Nucleic_Acid_QC_Result & varchar(5000) & & \\
\hline Average_Insert_Size_basepairs & float $(7,2)$ & & \\
\hline Sequencing_Provider_req & $\operatorname{varchar}(100)$ & & \\
\hline Date_at_Sequencing_Provider_req & date & & \\
\hline Sent_By_req & $\operatorname{varchar}(100)$ & & \\
\hline Experimentalist_Informatician_notes_upload & $\operatorname{varchar}(500)$ & & \\
\hline fileuploaded & mediumblob & & \\
\hline filetype & $\operatorname{varchar}(50)$ & & \\
\hline createdby & varchar(20) & & $x$ \\
\hline timecreation & timestamp & & $x$ \\
\hline modifiedby & varchar(20) & & $x$ \\
\hline timemodified & timestamp & & $x$ \\
\hline track_id & $\operatorname{int}(11)$ & & $x$ \\
\hline track_sample & $\operatorname{int}(11)$ & & $x$ \\
\hline
\end{tabular}

Table 5: Sequence MetaInformation

\begin{tabular}{|c|c|c|c|c|}
\hline Field Name & Data Type & Required Field & Automated Field & Drop-down Menu \\
\hline Experiment_Name_req & $\operatorname{varchar}(50)$ & & $x$ & \\
\hline Specimen_UID_req & $\operatorname{varchar}(100)$ & $x$ & & $x$ \\
\hline Specimen_UID_sec & $\operatorname{varchar}(50)$ & & & \\
\hline Sample_UID_req & $\operatorname{varchar}(100)$ & $x$ & & $x$ \\
\hline Sample_Alias_req & $\operatorname{varchar}(100)$ & $x$ & & $x$ \\
\hline SequenceRun_ID_req & $\operatorname{varchar}(100)$ & & $x$ & \\
\hline Sequence_Lane_req & $\operatorname{int}(11)$ & & & \\
\hline Sequence_Data & $\operatorname{varchar}(200)$ & & $x$ & \\
\hline Sequence_Data_Point & $\operatorname{varchar}(200)$ & & $x$ & \\
\hline Date_Sample_Submitted & date & & & \\
\hline Sequencing_Provider & $\operatorname{varchar}(100)$ & $x$ & & $x$ \\
\hline Sequencing_Platform & varchar(100) & $x$ & & $x$ \\
\hline ProviderSeqDirName & $\operatorname{varchar}(200)$ & & & \\
\hline fastqMatePair1 & $\operatorname{varchar}(50)$ & & & \\
\hline fastqMatePair2 & $\operatorname{varchar}(50)$ & & & \\
\hline
\end{tabular}




\begin{tabular}{|c|c|c|c|c|}
\hline ProjectSeqDirName & $\operatorname{varchar}(200)$ & & & \\
\hline Project & $\operatorname{varchar}(50)$ & $x$ & & $x$ \\
\hline Received & $\operatorname{varchar}(3)$ & & $x$ & \\
\hline Run_Plexing & $\operatorname{varchar}(100)$ & & & \\
\hline Primer_Set & $\operatorname{varchar}(30)$ & & & \\
\hline Barcode_Type & $\operatorname{varchar}(20)$ & & & \\
\hline Barcode & $\operatorname{varchar}(200)$ & & & \\
\hline Library_Preparation_ID_req & $\operatorname{varchar}(100)$ & & & \\
\hline Primer_Name & $\operatorname{varchar}(200)$ & & & \\
\hline Primer_Seq & $\operatorname{varchar}(200)$ & & & \\
\hline Date_Run_Performed & date & & & \\
\hline Run_Number & $\operatorname{int}(11)$ & & & \\
\hline Date_Data_Received & date & & & \\
\hline Total_Reads_Millions & float $(7,2)$ & & & \\
\hline Read_Type & $\operatorname{varchar}(100)$ & & & $x$ \\
\hline Read_Length_Bases & $\operatorname{int}(11)$ & & & $x$ \\
\hline Total_Bases_Billions & $\operatorname{int}(11)$ & & & \\
\hline $\begin{array}{l}\text { Experimentalists_notes_uploa } \\
\mathrm{d}\end{array}$ & $\operatorname{varchar}(100)$ & & & \\
\hline fileuploaded & mediumblob & & & \\
\hline filetype & $\operatorname{varchar}(50)$ & & & \\
\hline Remarks & $\operatorname{varchar}(100)$ & & & \\
\hline Server_Results_Folder & $\operatorname{varchar}(100)$ & & & \\
\hline createdby & $\operatorname{varchar}(20)$ & & $x$ & \\
\hline timecreation & timestamp & & $X$ & \\
\hline modifiedby & $\operatorname{varchar}(20)$ & & $x$ & \\
\hline timemodified & timestamp & & $X$ & \\
\hline track_id & $\operatorname{int}(11)$ & & $x$ & \\
\hline track_sequence & $\operatorname{int}(11)$ & & $x$ & \\
\hline track_exp & $\operatorname{int}(11)$ & & $x$ & \\
\hline
\end{tabular}

